\title{
Canada ranks low on patient safety in international comparison
}

$\mathrm{T}$ here is a "clear opportunity for improvement" on patient safety in Canada, according to the Canadian Institute for Health Information (CIHI).

Canada ranks below the Organisation for Economic Co-operation Development (OECD) average on several patient safety indicators, states the institute's new report Benchmarking Canada's Health System: International Comparisons.

In particular, Canada is among the worst for leaving objects such as sponges or medical equipment in patients after surgery. Obstetric trauma was also cited as a problem area. In instrument-assisted vaginal deliveries, $17 \%$ of women experienced a perineum tear, a higher rate than in all peer countries (those with comparable economies and health systems).

One reason for the low rankings, however, may be that Canada is better at collecting patient safety data than many other countries. Accuracy of sta- tistics can vary widely depending on how they are recorded and collected.

It is "not clear how we measure up internationally, but it is clear that we need to improve our own system," says Katerina Gapanenko, manager of health system research at CIHI.

Another area of concern highlighted in the report was Canada's lung cancer mortality rate of 58 deaths per 100000 people, compared to the OECD average of 43 deaths. Though the percentage of daily smokers $(16 \%)$ is actually lower than in most OECD nations, cancer can take years to develop and Canada is now seeing the effects of higher smoking rates from previous decades, explains Gapanenko.

"Lung cancer kills more than any other cancer," says Gapaneko. "More people die from lung cancer than prostate, breast and colorectal cancer combined."

Obesity is also a growing problem in Canada. The adult obesity rate $(17.7 \%)$ is second only to the United States (28.5\%) among peer countries. Canada performs equally poorly in this area among youth, with a quarter of children aged 5-17 considered overweight or obese.

Conversely, Canada performed well in the consumption of fruits and vegetables, especially in children. One possible explanation is that many Canadian school boards have banned certain unhealthy snacks from vending machines to try to improve students' eating habits.

Other areas in which Canada performs well include breast cancer screening, stroke mortality rates and admission rates to hospitals for asthma and diabetes, according to the report. "This means that we are doing better than others [in these areas], but it does not mean we don't have room to improve," says Gapaneko.

CIHI plans to release another report on quality of care in January 2014 that will place more emphasis on patient safety indicators. - Christina Pellegrini, CMAJ

CMAJ 2014. DOI:10.1503/cmaj.109-4665 